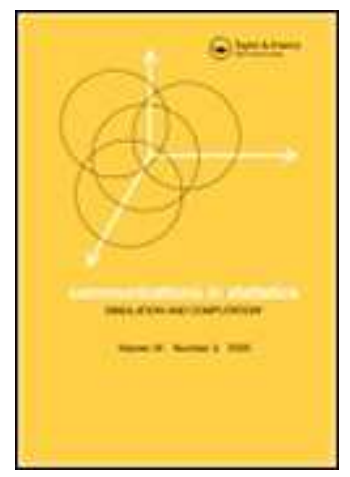

\title{
Kendall's $\mathbf{W}$ reconsidered
}

\begin{tabular}{|c|c|}
\hline Journal: & Communications in Statistics - Simulation and Computation \\
\hline Manuscript ID: & LSSP-2010-0099.R1 \\
\hline Manuscript Type: & Original Paper \\
\hline $\begin{array}{r}\text { Date Submitted by the } \\
\text { Author: }\end{array}$ & $18-O c t-2010$ \\
\hline Complete List of Authors: & $\begin{array}{l}\text { Grothe, Oliver; University of Cologne, Department of Economic and } \\
\text { Social Statistics } \\
\text { Schmid, Friedrich; University of Cologne, Department of Economic } \\
\text { and Social Statistics }\end{array}$ \\
\hline Keywords: & $\begin{array}{l}\text { Ranking, Empirical Copula Process, Comonotonicity, Jackknife, } \\
\text { Bootstrap, Monte Carlo Simulation }\end{array}$ \\
\hline Abstract: & $\begin{array}{l}\text { In their seminal paper, Kendall and Babington Smith (1939) } \\
\text { suggested a measure W to quantify the agreement between d } \\
\text { rankings of n objects. Its distribution was essentially investigated } \\
\text { under the assumption of independent rankings. In many } \\
\text { applications, however, the rankings are not independent. This paper } \\
\text { reconsiders Kendall's W, investigating its distribution for dependent } \\
\text { rankings using copula theory. We show that Kendall's W is } \\
\text { asymptotically normally distributed under very weak assumptions } \\
\text { and that its variance can be estimated by means of bootstrap and } \\
\text { jackknife. We present an application of Kendall's W to returns and } \\
\text { volatilities of the German DAX-30 assets. }\end{array}$ \\
\hline \multicolumn{2}{|c|}{$\begin{array}{l}\text { Note: The following files were submitted by the author for peer review, but cannot be converted } \\
\text { to PDF. You must view these files (e.g. movies) online. }\end{array}$} \\
\hline Gro_Schmi_W_ComStat.zip & \\
\hline
\end{tabular}




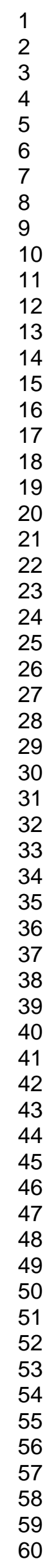

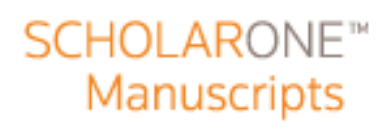

10

12

13

14

15

16

18

19

20

22

23

25

26

27

29

30

33

34

35

36

37

40

41

42

44

45

46

47

48

50

51

52

53

54

55

57

58

59

60

URL: http://mc.manuscriptcentral.com/Issp E-mail: comstat@univmail.cis.mcmaster.ca 
Kendall's $\mathcal{W}$ reconsidered

Oliver Grothe and Friedrich Schmid

Department of Economic and Social Statistics

University of Cologne

Albertus-Magnus-Platz, D-50923 Cologne

grothe@statistik.uni-koeln.de, schmid@wiso.uni-koeln.de

Key Words: Ranking, Rank Correlation, Spearman's rho, Comonotonicity, Independence, Copula, Empirical Copula Process, Bootstrap, Jackknife, Monte Carlo Simulation.

\begin{abstract}
In their seminal paper, Kendall and Babington Smith (1939) suggested a measure $\mathcal{W}$ to quantify the agreement between $d$ rankings of $n$ objects. Its distribution was essentially investigated under the assumption of independent rankings. In many applications, however, the rankings are not independent. This paper reconsiders Kendall's $\mathcal{W}$, investigating its distribution for dependent rankings using copula theory. We show that Kendall's $\mathcal{W}$ is asymptotically normally distributed under very weak assumptions and that its variance can be estimated by means of bootstrap and jackknife. We present an application of Kendall's $\mathcal{W}$ to returns and volatilities of the German DAX-30 assets.
\end{abstract}

\title{
1 Introduction
}

If $n$ objects are ranked by $d$ individuals according to some property, the question arises whether the $d$ rankings show any agreement or are more or less independent. In their seminal paper, Kendall and Babington Smith (1939) suggested a measure $\mathcal{W}$ (now known as Kendall's $\mathcal{W})$ to quantify the strength of agreement. It was defined in such way that $0 \leq \mathcal{W} \leq 1$ and $\mathcal{W}=1$ if and only if the $d$ rankings are identical. Kendall's $\mathcal{W}$ was essentially used as a test statistic to test for the null hypothesis of $d$ independent rankings. For this reason its distribution was investigated under the assumption of independence, usually called the null 
This paper argues, however, that $\mathcal{W}$ is not a suitable test statistic for testing the independence of $d$ rankings. It can be shown that $\mathcal{W}$ is based on pairwise rank correlation and therefore can only test against pairwise independence. Moreover, testing the independence of $d$ rankings, where $d \geq 2$, has been intensively considered in the literature and powerful tests have been suggested. Recent contributions, for instance, include Genest and Rémillard (2004) and Genest et al. (2007).

Instead of testing for independence, Kendall's $\mathcal{W}$ is a useful statistic to measure the strength of agreement of the $d$ rankings or the closeness of the $d$ rankings to perfect agreement, i.e., to comonotonicity. For this, however, it is crucial to know the distribution of $\mathcal{W}$ in the non-null case, i.e., in the case where the rankings are not independent. This paper reconsiders Kendall's $\mathcal{W}$ under this aspect. Former literature addressing the non-null case includes only some special results (see, e.g., Mallows (1957), Loretta and Schucany (1975), Kraemer (1976), Palachek and Schucany (1984)). A major problem seems to be the definition of a proper population version (or theoretical version) for $\mathcal{W}$ in the non-null case.

The present paper uses copula theory for this purpose. A copula can be viewed as a continuous version of a table of $d$ rankings of $n$ objects, and thus the copula approach seems to be the most general approach for the definition of $\mathcal{W}$. The use of copulas entails the assumption, that the $d$ rankings are based on $d$ latent continuous variables $X_{1}, \ldots, X_{d}$ with copula $C$. Observations of $X_{1}, \ldots, X_{d}$, however, are not required and the procedures suggested are solely based on the original table of ranks. Using results on the so-called empirical copula process, it can be shown that the normalized empirical version of Kendall's $\mathcal{W}$ is asymptotically normally distributed under very weak assumptions on the basic copula and that a closed formula for its asymptotic variance exists.

Asymptotic normality of Kendall's $\mathcal{W}$ in the non-null case makes it possible to construct confidence intervals or hypothesis tests for the strength of agreement of the $d$ rankings, which might be useful in empirical applications. The crucial point is the determination of the standard deviation of Kendall's $\mathcal{W}$ which is possible by using resampling methods such 
as the bootstrap or the jackknife.

The structure of the paper is as follows: Section 2 introduces notations and some basic concepts of copula theory and statistics to be used in further sections. Subsection 3.1 proposes a population version of Kendall's $\mathcal{W}$ using copulas. In subsection 3.2, the empirical version of Kendall's $\mathcal{W}$ is shown to be asymptotically normally distributed under very weak assumptions on the copula. Subsection 3.3 discusses variance estimation for Kendall's $\mathcal{W}$ using the jackknife and the bootstrap. A simulation study based on the Gaussian and Clayton families of copulas investigates the performance of these resampling methods in small- and medium-sized samples. Subsection 3.4 explores the goodness of fit of normal approximations to Kendall's $\mathcal{W}$ for the above stated families of copulas. In an empirical example, section 4 applies Kendall's $\mathcal{W}$ to measure the strength of association of returns and volatilities of the 30 assets of the German DAX index. Section 5 concludes.

\section{Notation and definitions}

Throughout the paper we assume that $\mathbf{X}=\left(X_{1}, \ldots, X_{d}\right)$, where $d \geq 2$, is a random vector with continuous distribution function

$$
F(\mathbf{x})=P\left(X_{1} \leq x_{1}, \ldots, X_{d} \leq x_{d}\right), \mathbf{x}=\left(x_{1}, \ldots, x_{d}\right) \in \mathbb{R}^{d}
$$

and continuous marginal distribution functions $F_{i}\left(x_{i}\right)=P\left(X_{i} \leq x_{i}\right)$ for $x_{i} \in \mathbb{R}$ and $i=$ $1, \ldots, d$. Thus, according to Sklar's (1959) theorem, a unique copula

$$
C:[0,1]^{d} \rightarrow[0,1]
$$

exists such that

$$
F(\mathbf{x})=C\left(F_{1}\left(x_{1}\right), \ldots, F_{d}\left(x_{d}\right)\right) \text { for } \mathbf{x} \in \mathbb{R}^{d}
$$

The copula $C$ is the joint distribution function of the random vector

$$
\mathbf{U}=\left(U_{1}, \ldots, U_{d}\right)=\left(F_{1}\left(X_{1}\right), \ldots, F_{d}\left(X_{d}\right)\right)
$$


i.e.,

$$
C\left(u_{1}, \ldots, u_{d}\right):=P\left(U_{1} \leq u_{1}, \ldots, U_{d} \leq u_{d}\right) \text { for } \mathbf{u}=\left(u_{1}, \ldots, u_{d}\right) \in[0,1]^{d} .
$$

The univariate margins $U_{i}$ are uniformly distributed on $[0,1]$.

A detailed treatment of copulas is given in Joe (1997), Cherubini et al. (2004) and Nelsen (2006). An important example of a parametric family of copulas is the Gaussian copula (see Joe (1997)) which will be used in the subsequent sections. It is defined by

$$
C_{\Theta}\left(u_{1}, \ldots, u_{d}\right)=\Phi_{\Theta}\left(\Phi^{-1}\left(u_{1}\right), \ldots, \Phi^{-1}\left(u_{d}\right)\right)
$$

where $\Phi_{\Theta}$ is the distribution function of a multivariate normal distribution with mean zero, unit variances and correlation matrix $\boldsymbol{\Theta}=\left(\theta_{i j}\right)_{i, j=1 \ldots d} . \Phi^{-1}$ denotes the quantile function of a standard normal distribution.

Another example for a parametric family of copulas is the Clayton family (see Clayton (1978)), given by

$$
C_{\theta}\left(u_{1}, \ldots, u_{d}\right):=\left(\sum_{i=1}^{d} u_{i}^{-\theta}-(d-1)\right)^{-1 / \theta}
$$

where $\theta>0$.

It is well known that every copula $C$ is bounded in the following sense

$$
\begin{aligned}
W(\mathbf{u}) & :=\max \left\{u_{1}+\ldots+u_{d}-(d-1), 0\right\} \\
& \leq C(\mathbf{u}) \leq \min \left\{u_{1}, \ldots, u_{d}\right\}=: M(\mathbf{u}), \text { for } \mathbf{u} \in[0,1]^{d},
\end{aligned}
$$

where $M$ and $W$ are called the upper and lower Frechet-Hoeffding bounds, respectively. The lower bound $W$ is a copula itself only if $d=2$. The upper bound $M=\min \left\{u_{1}, \ldots, u_{d}\right\}$ is always a copula and is called comonotonic copula. A random vector $\mathbf{X}$ with copula $M$ is called comonotonic. Comonotonicity is a pairwise property: $\mathbf{X}=\left(X_{1}, \ldots, X_{d}\right)$ is comonotonic if and only if any pair $\left(X_{k}, X_{l}\right)$ is comonotonic, where $k \neq l$ and $k, l \in\{1, \ldots, d\}$. For a proof see Dhaene et al. (2000a).

Another important copula is the independence copula

$$
\Pi(\mathbf{u})=\prod_{i=1}^{d} u_{i}, \quad \mathbf{u} \in[0,1]^{d} .
$$


It is the copula of a vector $\mathbf{X}$ of independent random variables $X_{1}, \ldots, X_{d}$. Contrary to comonotonicity, independence is not a pairwise property. Pairwise independence of $X_{k}$ and $X_{l}$ for $k<l$ and $k, l \in\{1, \ldots, d\}$ doesn't entail independence of $\mathbf{X}$. For example, consider a $d$-variate Farlie-Gumbel-Morgenstern copula,

$$
C\left(u_{1}, \ldots, u_{d}\right)=\prod_{i=1}^{d} u_{i}+\theta\left(\prod_{i=1}^{d} u_{i}\left(1-u_{i}\right)\right),|\theta| \leq 1 .
$$

For $\theta \neq 0$ the copula $C$ is not the independence copula but the copulas of its $d^{*}<d$ dimensional margins are independence copulas.

Gaussian and Clayton copulas interpolate the comonotonic and the independence copula: For the Gaussian copula $C_{\boldsymbol{\Theta}}(\mathbf{u})=\Pi(\mathbf{u})$ if and only if $\Theta=\mathbf{I}$. For the case of equicorrelation, i.e., $\boldsymbol{\Theta}=(1-\theta) \mathbf{I}+\theta \mathbf{1 1}^{\prime}$ and $-\frac{1}{d-1}<\theta<1$, it can be shown that

$$
\lim _{\theta \rightarrow 1} C_{(1-\theta) \mathbf{I}+\theta 11^{\prime}}(\mathbf{u})=M(\mathbf{u}) \text { for } \mathbf{u} \in[0,1]^{d}
$$

For the Clayton copula we have

$$
\lim _{\theta \rightarrow 0} C_{\theta}(\mathbf{u})=\Pi(\mathbf{u}) \text { and } \lim _{\theta \rightarrow \infty} C_{\theta}(\mathbf{u})=M(\mathbf{u}) \text { for } \mathbf{u} \in[0,1]^{d}
$$

A well known measure of bivariate association of two random variables is Spearman's rho, $\rho_{S}$. For $X_{k}$ and $X_{l}$ it is

$$
\begin{aligned}
\rho_{S, k l} & =\frac{\operatorname{cov}\left(F_{k}\left(X_{k}\right), F_{l}\left(X_{l}\right)\right)}{\sqrt{\operatorname{var}\left(F_{k}\left(X_{k}\right)\right)} \sqrt{\operatorname{var}\left(F_{l}\left(X_{l}\right)\right)}} \\
& =\frac{\operatorname{cov}\left(U_{k}, U_{l}\right)}{\sqrt{\operatorname{var}\left(U_{k}\right)} \sqrt{\operatorname{var}\left(U_{l}\right)}}=12 \int_{0}^{1} \int_{0}^{1} C_{k l}\left(u_{k}, u_{l}\right) d u_{k} d u_{l}-3,
\end{aligned}
$$

where $C_{k l}\left(u_{k}, u_{l}\right)=C\left(1, \ldots, 1, u_{k}, 1, \ldots, 1, u_{l}, 1, \ldots, 1\right)$ denotes the marginal copula of $X_{k}$ and $X_{l}$. It can be seen that $\rho_{S, k l}$ depends on the copula $C_{k l}$ only, and is invariant with respect to the marginal distributions of $X_{k}$ and $X_{l}$. It is $\rho_{S, k l}=1$ if and only if $C_{k l}=\min \left\{u_{k}, u_{l}\right\}$ and therefore $\rho_{S, k l}=1$ if and only if $X_{k}$ and $X_{l}$ are comonotonic, see Nelsen (2006). Using that comonotonicity is a pairwise property, it follows that $\mathbf{X}=\left(X_{1}, \ldots, X_{d}\right)$ is comonotonic if and only if every $\rho_{S, k l}=1$, where $k<l$ and $k, l \in\{1, \ldots, d\}$. 
In contrast, $\rho_{S, k l}=0$ does not imply independence of $X_{k}$ and $X_{l}$, and thus $\rho_{S, k l}=0$ for $k<l$ and $k, l \in\{1, \ldots, d\}$ does not imply that $\mathbf{X}=\left(X_{1}, \ldots, X_{d}\right)$ has independent components.

Let $\mathbf{X}_{1}, \mathbf{X}_{2}, \ldots, \mathbf{X}_{n}$ be a sequence of i.i.d. observations on $\mathbf{X}$, where

$$
\mathbf{X}_{j}=\left(X_{1 j}, \ldots, X_{d j}\right)^{\prime}
$$

for $j=1 \ldots, n$ and let

$$
\widehat{F}_{i, n}(x)=\frac{1}{n} \sum_{j=1}^{n} 1_{\left\{X_{i j} \leq x\right\}}
$$

be the empirical distribution function for $i=1, \ldots, d$ and $x \in \mathbb{R}$.

Further, set

$$
\widehat{U}_{i j, n}:=\widehat{F}_{i, n}\left(X_{i j}\right)
$$

for $i=1, \ldots, d$ and $j=1, \ldots, n$. Note that

$$
\widehat{U}_{i j, n}=\frac{1}{n}\left(\operatorname{rank} \text { of } X_{i j} \text { in } X_{i 1}, \ldots, X_{i n}\right)=\frac{1}{n} R_{i j, n} .
$$

Let

$$
\widehat{\mathbf{U}}_{j, n}=\left(\widehat{U}_{1 j, n}, \ldots, \widehat{U}_{d j, n}\right)^{\prime}
$$

for $j=1, \ldots, n$ and let

$$
\mathbf{R}=\left(\mathbf{R}_{1, n}, \ldots, \mathbf{R}_{n, n}\right)=\left(n \widehat{\mathbf{U}}_{1, n}, \ldots, n \widehat{\mathbf{U}}_{n, n}\right)
$$

denote the corresponding $d \times n$ table of ranks. Statistical inference for Kendall's $\mathcal{W}$ in the following sections is entirely based on $\mathbf{R}$. Knowledge of the latent variables $\mathbf{X}_{1}, \mathbf{X}_{2}, \ldots, \mathbf{X}_{n}$ is not required.

\section{Kendall's $\mathcal{W}$}

This section reconsiders Kendall's $\mathcal{W}$ and its asymptotic and finite sample distribution. In order to investigate distributional issues about Kendall's $\mathcal{W}$, we must have a population 
version, i.e., a theoretical version of Kendall's $\mathcal{W}$ which depends on the distribution of the basic random vector $\mathbf{X}=\left(X_{1}, \ldots, X_{d}\right)$ via its copula, but does not depend on the marginal distribution of $X_{i}, i=1, \ldots, d$. The following subsection 3.1 considers such a version of Kendall's $\mathcal{W}$.

\subsection{A population version of Kendall's $\mathcal{W}$}

Let $\mathbf{X}=\left(X_{1}, \ldots, X_{d}\right)$ denote the basic random vector with continuous marginal distribution functions $F_{i}$ and uniquely determined copula $C$, i.e.,

$$
\begin{aligned}
C\left(u_{1}, \ldots, u_{d}\right) & =P\left(U_{1} \leq u_{1}, \ldots, U_{d} \leq u_{d}\right) \\
& =P\left(F_{1}\left(X_{1}\right) \leq u_{1}, \ldots, F_{d}\left(X_{d}\right) \leq u_{d}\right)
\end{aligned}
$$

for $\mathbf{u}=\left(u_{1}, \ldots, u_{d}\right) \in[0,1]^{d}$. We consider the following variable

$$
U:=\sum_{i=1}^{d} U_{i}=\sum_{i=1}^{d} F_{i}\left(X_{i}\right)
$$

Obviously, $\mathrm{E}_{C}(U)=d / 2$ for every copula $C$. The variance of $U$, however, depends on $C$. If $C=\Pi$ (i.e., the variables $X_{1}, \ldots, X_{d}$ are independent), we have:

$$
\operatorname{var}_{\Pi}(U)=\frac{1}{12} \cdot d
$$

If $C=M$ (i.e., the variables $X_{1}, \ldots, X_{d}$ are comonotonic), we have:

$$
\operatorname{var}_{M}(U)=\operatorname{var}\left(d \cdot U_{1}\right)=\frac{1}{12} \cdot d^{2}
$$

The value $d^{2} / 12$ is an upper bound for $\operatorname{var}_{C}(U)$ for arbitrary $C$. A lower bound is $\operatorname{var}_{C}(U)=0$. We look for examples of copulas $C$ where $\operatorname{var}_{C}(U)=0$.

1. For $d=2$ let $C\left(u_{1}, u_{2}\right)=W\left(u_{1}, u_{2}\right)$. Then $U_{2}=1-U_{1}$ and $U=U_{1}+U_{2}=1=$ const. Thus $\operatorname{var}_{C}(U)=0$. Related examples can be constructed for $d>2$. For $d=4$, for example, the copula $C\left(u_{1}, u_{2}, u_{3}, u_{4}\right)=W\left(u_{1}, u_{2}\right) W\left(u_{3}, u_{4}\right) \operatorname{has}_{\operatorname{var}_{C}}(U)=0$. 
2. For $d \geq 2$ let $X_{1}, \ldots, X_{d}$ be such, that the corresponding $U_{1}=F_{1}\left(X_{1}\right), \ldots, U_{d}=$ $F_{d}\left(X_{d}\right)$ are equicorrelated with

$$
\operatorname{corr}\left(U_{i}, U_{j}\right)=-\frac{1}{d-1}
$$

Then

$$
\operatorname{var}(U)=\frac{1}{12} d+d(d-1)\left(-\frac{1}{d-1}\right) \cdot \frac{1}{12}=0 .
$$

We now define $\mathcal{W}_{0}$ by normalizing $\operatorname{var}(U)$ :

$$
\mathcal{W}_{0}:=\frac{\operatorname{var}(U)}{d^{2} / 12}=\frac{12 \operatorname{var}(U)}{d^{2}}
$$

From the upper and lower bounds of $\operatorname{var}(U)$, we get

$$
0 \leq \mathcal{W}_{0} \leq 1
$$

Further, $\mathcal{W}_{0}=1 / d$ if $\mathbf{U}=\left(U_{1}, \ldots, U_{d}\right)$ is independent. However, as discussed above, $\mathcal{W}_{0}=$ $1 / d$ does not imply the independence of $\mathbf{U}$.

$\mathcal{W}_{0}$ is a population version of Kendall's $\mathcal{W}$. It is closely related to the mean $\bar{\rho}_{S}$ of $d(d-1) / 2$ pairwise Spearman's rho, $\rho_{S, k l}$, of $U_{k}$ and $U_{l}$.

Indeed

$$
\begin{aligned}
\frac{\operatorname{var}(U)-d / 12}{d^{2} / 12-d / 12} & =\frac{1}{d(d-1)}(12 \operatorname{var}(U)-d) \\
& =\frac{1}{d(d-1)}\left(12\left(\sum_{i=1}^{d} \operatorname{var}\left(U_{i}\right)+\sum_{k \neq l} \operatorname{cov}\left(U_{k}, U_{l}\right)\right)-d\right) \\
& =\frac{1}{d(d-1)}\left(12 \frac{d}{12}+12 \sum_{k \neq l} \operatorname{cov}\left(U_{k}, U_{l}\right)-d\right) \\
& =\frac{1}{d(d-1)}\left(12 \sum_{k \neq l} \operatorname{cov}\left(U_{k}, U_{l}\right)\right)^{-1} \\
& =\left(\begin{array}{l}
d \\
2
\end{array}\right)^{-1} \sum_{k<l} \operatorname{corr}\left(U_{k}, U_{l}\right)=\left(\begin{array}{l}
d \\
2
\end{array}\right)^{-1} \sum_{k<l} \rho_{S, k l}=\bar{\rho}_{S},
\end{aligned}
$$


where $\rho_{S, k l}=\operatorname{corr}\left(U_{k}, U_{l}\right)$ is the population version of Spearman's rho of $X_{i}$ and $X_{j}$. We thus have

$$
\mathcal{W}_{0}=\frac{(d-1) \cdot \bar{\rho}_{S}+1}{d}
$$

and

$$
\bar{\rho}_{S}=\frac{d \mathcal{W}_{0}-1}{d-1}
$$

In the following, we provide examples of $\mathcal{W}_{0}$ and $\bar{\rho}_{S}$ for some special copulas $C$ described in section 2 ,

- If $C=\Pi$ then $\bar{\rho}_{S}=0$ and $\mathcal{W}_{0}=1 / d$. Note that the converse is not true. For example, for the Farlie-Gumbel-Morgenstern copula mentioned above, all pairwise Spearman's rho are equal to zero and therefore $\mathcal{W}_{0}=1 / d$ although $C$ is not the independence copula.

- $\mathcal{W}_{0}=\bar{\rho}_{S}=1$ if and only if $C=M$, i.e., $\mathcal{W}_{0}=\bar{\rho}_{S}=1$ if and only if $\mathbf{X}=\left(X_{1}, \ldots, X_{d}\right)$ is comonotonic. This is directly derived from the fact that $\bar{\rho}_{S}=1$ if and only if $\rho_{S, k l}=1$ for $k, l=1, \ldots, n$ and $k \neq l$, because this is equivalent to the comonotonicity of $\mathbf{X}=\left(X_{1}, \ldots, X_{d}\right)$.

- If $C=C_{\boldsymbol{\Theta}}$, a $d$-dimensional Gaussian copula with parameter matrix $\boldsymbol{\Theta}=\left(\theta_{i j}\right), i, j=$ $1 \ldots d$, then

$$
\bar{\rho}_{S}=\frac{6}{\pi}\left(\begin{array}{l}
d \\
2
\end{array}\right)^{-1} \sum_{i<j} \arcsin \left(\theta_{i j} / 2\right)
$$

(see Embrechts et al. (2002)) and

$$
\mathcal{W}_{0}=\frac{12}{\pi d^{2}} \sum_{i<j} \arcsin \left(\theta_{i j} / 2\right)+\frac{1}{d}
$$

In the case of equicorrelation, i.e., if $\theta_{i j}=\theta$ for $i \neq j$ and $\frac{-1}{d-1} \leq \theta \leq 1$, we have

$$
\mathcal{W}_{0}=\left(\frac{d-1}{d}\right) \frac{6}{\pi} \arcsin (\theta / 2)+\frac{1}{d} \text {. }
$$

- In case of the Clayton copula a simple formula to express Spearman's rho as a function of $\theta$ is not available. Numerical evaluations, however, are possible (see Joe (1997)). 


\subsection{Empirical version of Kendall's $\mathcal{W}$ and its asymptotic distribu- tion}

This section introduces empirical versions, i.e., estimators, of $\mathcal{W}_{0}$ and $\bar{\rho}_{S}$. The estimators are based on ranks given by the table $\mathbf{R}$ as defined in section 2. It is assumed that the joint distribution function $F$, the copula $C$ and the marginal distribution functions $F_{i}$ are all unknown.

A natural estimator for $\bar{\rho}_{S}=\left(\begin{array}{l}d \\ 2\end{array}\right)^{-1} \sum_{k<l} \rho_{S, k l}$ is

$$
\widehat{\bar{\rho}}_{S, n}=\left(\begin{array}{l}
d \\
2
\end{array}\right)^{-1} \sum_{k<l} \widehat{\rho}_{S, k l, n},
$$

where

$$
\begin{aligned}
\widehat{\rho}_{S, k l, n} & =\frac{\sum_{j=1}^{n}\left(R_{k j, n}-\frac{n(n+1)}{2}\right)\left(R_{l j, n}-\frac{n(n+1)}{2}\right)}{\frac{n^{3}-n}{12}} \\
& =\frac{\sum_{j=1}^{n}\left(\widehat{U}_{k j, n}-\frac{n+1}{2}\right)\left(\widehat{U}_{l j, n}-\frac{n+1}{2}\right)}{\frac{n^{3}-n}{12 n^{2}}} .
\end{aligned}
$$

To estimate $\mathcal{W}_{0}$ let

$$
\widehat{U}_{\cdot j, n}=\sum_{i=1}^{d} \widehat{U}_{i j, n}
$$

with mean

$$
\frac{1}{n} \sum_{j=1}^{n} \widehat{U}_{\cdot j, n}=\frac{1}{n} d \frac{n(n+1)}{2 n}=\frac{1}{n} d \frac{n+1}{2}
$$

and variance

$$
\frac{1}{n} \sum_{j=1}^{n}\left(\widehat{U}_{\cdot j, n}-d \frac{n+1}{2 n}\right)^{2}=\frac{\sum_{j=1}^{n}\left(n \widehat{U}_{\cdot j, n}-d \frac{n+1}{2}\right)^{2}}{n^{3}}
$$

In the case of comonotonicity of the ranks, we have $\widehat{U}_{\cdot j, n}=d / n \cdot j$ without loss of generality. The variance term above is then equal to

$$
\frac{d^{2}}{12}\left(1-\frac{1}{n^{2}}\right) \text {. }
$$


Therefore, an estimator of $\mathcal{W}_{0}$ is

$$
\widehat{\mathcal{W}}_{n}=\frac{12 \sum_{j=1}^{n}\left(n \widehat{U}_{\cdot j, n}-d \frac{n+1}{2}\right)^{2}}{d^{2}\left(n^{3}-n\right)}
$$

which coincides with the original Kendall statistic, Kendall's $\mathcal{W}$, suggested in Kendall and Babington Smith (1939).

It has been shown in Kendall and Gibbons (1990) that

$$
\widehat{\bar{\rho}}_{S, n}=\left(\begin{array}{l}
d \\
2
\end{array}\right)^{-1} \sum_{k<l} \widehat{\rho}_{S, k l, n}=\frac{d \widehat{\mathcal{W}}_{n}-1}{d-1}
$$

and

$$
\widehat{\mathcal{W}}_{n}=\frac{(d-1) \widehat{\bar{\rho}}_{S, n}+1}{d}
$$

which are the empirical counterparts to equations (2) and (3) in section 3.1.

The asymptotic distribution of $\widehat{\mathcal{W}}_{n}$ is established in the following theorem:

Theorem (Asymptotic normality of $\widehat{\mathcal{W}}_{n}$ ).

Let $F$ be a continuous d-dimensional distribution function with copula $C$. Under the additional assumption that the $i$-th partial derivatives $\mathrm{D}_{i} C(\mathbf{u})$ exist and are continuous for $i=1, \ldots, d$, we have

$$
\sqrt{n}\left(\widehat{\mathcal{W}}_{n}-\mathcal{W}_{0}\right) \stackrel{w}{\longrightarrow} Z \sim N\left(0, \sigma_{C}^{2}\right)
$$

where

$$
\sigma_{C}^{2}=144\left(\frac{d-1}{d}\right)^{2} \sum_{k<l, s<t}\left(\begin{array}{l}
d \\
2
\end{array}\right)^{-2} \int_{[0,1]^{2}} \int_{[0,1]^{2}} \mathrm{E}\left[\mathbb{G}_{C}\left(\mathbf{u}^{(k, l)}\right) \mathbb{G}_{C}\left(\mathbf{v}^{(s, t)}\right)\right] d v_{s} d v_{t} d u_{k} d u_{l}
$$

and $\stackrel{w}{\longrightarrow}$ denotes convergence in distribution. Further,

$$
\mathbb{G}_{C}(\mathbf{u})=\mathbb{B}_{C}(\mathbf{u})-\sum_{i=1}^{d} D_{i} C(\mathbf{u}) \mathbb{B}_{C}\left(\mathbf{u}^{(i)}\right)
$$

where $\mathbb{B}_{C}$ is a tight centered Gaussian process on $[0,1]^{d}$ with covariance function

$$
E\left(\mathbb{B}_{C}(\mathbf{u}) \mathbb{B}_{C}(\mathbf{v})\right)=C(\mathbf{u} \wedge \mathbf{v})-C(\mathbf{u}) C(\mathbf{v})
$$


and

$$
\begin{aligned}
\mathbf{u}^{(k, l)} & =\left(1, \ldots, 1, u_{k}, 1, \ldots, 1, u_{l}, 1, \ldots, 1\right), \\
\mathbf{u}^{(i)} & =\left(1, \ldots, 1, u_{i}, 1, \ldots, 1\right)
\end{aligned}
$$

Proof: See appendix.

Thus, $\widehat{W}_{n}$ is asymptotically normally distributed under weak assumptions concerning the basic copula $C$. Note that these assumptions exclude $C=M$, i.e., the case of comonotonicity. In the latter case it is easy to see that $\mathcal{W}_{0}=\widehat{\mathcal{W}}_{n} \equiv 1$ and the variance of $\widehat{\mathcal{W}}_{n}$ is zero.

The asymptotic variance $\sigma_{C}^{2}$ of $\widehat{\mathcal{W}}_{n}$ depends on the copula $C$ and, in general, cannot be written as a function of $\mathcal{W}_{0}$ as there are different copulas $C$ with the same $\mathcal{W}_{0}$.

However, for the cases $C=\Pi$ with $\mathcal{W}_{0}=\frac{1}{d}$, i.e., in the case of independence, it follows that

$$
\begin{aligned}
\sigma_{\Pi}^{2} & =\left(\frac{d-1}{d}\right)^{2}\left(\begin{array}{l}
d \\
2
\end{array}\right)^{-1} \\
& =\frac{2(d-1)}{d^{3}}=\frac{2}{d}\left(\frac{1}{d}\left(1-\frac{1}{d}\right)\right)
\end{aligned}
$$

and $\sigma_{\Pi}^{2}$ is a function of $d$ and $\mathcal{W}_{0}=1 / d$. The proof can be found in the appendix. The independence case, however, is not the focus of this paper. It would be interesting to know whether a similar formula for $\mathcal{W}_{0}$ can at least serve as an approximation to $\sigma_{C}^{2}$ in the general case.

\subsection{Variance estimation for Kendall's $\mathcal{W}$}

Although the theorem in section 3.2 yields an explicit formula for $\sigma_{C}^{2}$, its use in empirical applications is limited, as it relies on the unknown copula $C$ and is of extremely difficult structure. Reliable estimators for the variance or standard deviation of $\widehat{\mathcal{W}}_{n}$ can be obtained by resampling methods such as the bootstrap or the jackknife. For asymptotic justification of these methods see Shao and Dongsheng (1995). Note that these justifications are with respect to $n$ (i.e., $n \rightarrow \infty$ ) whereas $d$ is arbitrary but fixed. Concerning the 
bootstrap it is assumed (as in section 2) that the basic latent variables $\mathbf{X}_{1}, \ldots, \mathbf{X}_{n}$, are i.i.d. It has been shown that the bootstrap is asymptotically valid under this assumption (see Schmid and Schmidt (2007)).

The bootstrap is performed as follows. Let

$$
\left(n \widehat{\mathbf{U}}_{1, n}, \ldots, n \widehat{\mathbf{U}}_{n, n}\right)=\left(\mathbf{R}_{1, n}, \ldots, \mathbf{R}_{n, n}\right)=\mathbf{R}
$$

denote the $d \times n$ table of ranks as introduced in section 2, Let $\left(\mathbf{R}_{1, n}^{(b)}, \ldots, \mathbf{R}_{n, n}^{(b)}\right), b=1, \ldots, B$ denote the $b$-th bootstrap sample from $\left(\mathbf{R}_{1, n}, \ldots, \mathbf{R}_{n, n}\right)$. Each bootstrap sample is derived by sampling with replacement and reranking it in the case of ties, e.g., by using mid-ranks. The bootstrap standard deviation of $\widehat{\mathcal{W}}_{n}$ is then estimated by

$$
\widehat{\sigma}_{\widehat{\mathcal{W}}_{n}}^{B}=\sqrt{\frac{1}{B-1} \sum_{b=1}^{B}\left(\widehat{\mathcal{W}}_{n}^{(b)}-{\overline{\widehat{\mathcal{W}}_{n}}}^{2}\right.},
$$

where $\widehat{\mathcal{W}}_{n}^{(b)}$ is the estimate of Kendall's $\mathcal{W}$ based on the $b$-th bootstrap sample.

Like the bootstrap, the jackknife has to be done with respect to objects (i.e., by deleting a column of the table $\mathbf{R}$ ). Jackknifing with respect to the $d$ rankings (i.e., deleting a row in the table) changes the dependence structure of the $d$ rankings and leads to implausible results in simulations. For the jackknife estimate let

$$
\mathbf{R}_{-j}=\left(\mathbf{R}_{1, n}, \ldots, \mathbf{R}_{j-1, n}, \mathbf{R}_{j+1, n}, \ldots, \mathbf{R}_{n, n}\right)
$$

denote the $d \times(n-1)$ table obtained from $\mathbf{R}$ by deleting the $j$-th column $(j=1, \ldots, n)$. By $\widetilde{\mathbf{R}}_{-j}$ we denote the reranked table (with ranks from 1 to $(n-1)$ ).

If $\widehat{\mathcal{W}}_{n-1}^{(j)}$ denotes Kendall's $\mathcal{W}$ based on $\widetilde{\mathbf{R}}_{-j}$,

$$
\widehat{\sigma}_{\widehat{\mathcal{W}}_{n}}^{J}=\sqrt{\frac{n-1}{n} \sum_{j=1}^{n}\left(\widehat{\mathcal{W}}_{n-1}^{(j)}-\overline{\widehat{\mathcal{W}}}_{n-1}\right)^{2}}
$$

defines the jackknife estimate of the standard deviation of $\widehat{\mathcal{W}}_{n}$.

The properties of both estimators in finite samples are investigated by simulation. We consider the Gaussian copula (table 1) and the Clayton copula (table 2) with different dimensions $d=3,5,10$ and different parameters. The Gaussian copula is in general 
determined by $n(n-1) / 2$ correlation parameters. In order to reduce the number of parameters in our simulations, we have confined ourselves to the equicorrelation case. Simulations for more complex correlation structures have been conducted and the results may be obtained from the authors on request. We did not find, however, any fundamental differences to the case of equicorrelation. Contrary to the Gaussian copula, the Clayton copula has lower tail dependence. Therefore, the Clayton copula shows strong association for low ranks. An association structure like that may well occur in practice. Think of referees showing strong agreement for the best ranked candidates but weak for the others. The sample sizes selected are $n=5,10,20,50,100 . \mathcal{W}_{0}$ denotes the true value of Kendall's $\mathcal{W}$ for the parameters considered and $m($.$) and s($.$) denote the mean and standard deviation of$ the respective quantity over $N=10000$ Monte Carlo replications. Each bootstrap estimator relies on $B=500$ bootstrap samples.

The interpretation of the simulation results is straightforward. Comparing column $m\left(\widehat{\mathcal{W}}_{n}\right)$ and column $\mathcal{W}_{0}$ in tables 1 and 2 proves that there is some bias in $\widehat{\mathcal{W}}_{n}$ for $n=5,10$ and 20, in particular when the value of $\mathcal{W}_{0}$ is near 0 and 1 . Comparing column $s\left(\widehat{\mathcal{W}}_{n}\right)$ with $m\left(\widehat{\sigma}^{B}\right)$ and $m\left(\widehat{\sigma}^{J}\right)$ shows that the bootstrap and the jackknife estimates are reliable for the standard deviation of $\widehat{\mathcal{W}}_{n}$ for $n \geq 20$ and that there is no noticeable difference between the two estimates. Even for $n=5$ and 10 , none is superior to the other.

Comparing the standard deviations of both methods, i.e., columns $s\left(\widehat{\sigma}^{B}\right)$ and $s\left(\widehat{\sigma}^{J}\right)$, it can be seen that $s\left(\widehat{\sigma}^{B}\right)$ is often smaller than $s\left(\widehat{\sigma}^{J}\right)$ for $n=5$ and 10. For $n \geq 20$ there is no noticeable difference. Note, however, that the Jackknife is computationally more efficient for the considered sample sizes.

The simulated and the asymptotic standard deviation of $\widehat{\mathcal{W}}_{n}$ can be compared in the case of independence (i.e., $\theta=0$ for the Gaussian copula). Comparing $s\left(\widehat{\mathcal{W}}_{n}\right)$ in table 1 with $\sigma_{\Pi} / \sqrt{n}$ (see end of section 3.2 ) shows good agreement. The deviations are smaller than 0.01 for $n \geq 10$. 


\section{$\underline{\mathrm{CP}^{B} \quad \mathrm{CP}^{J}}$}

Dimension $d=3$

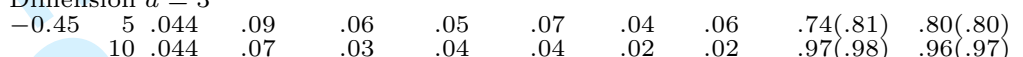

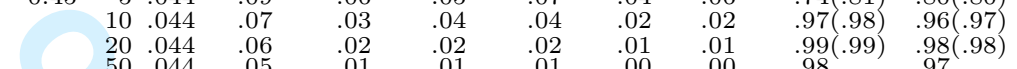

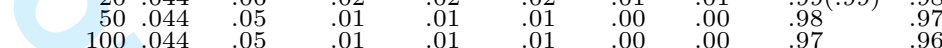

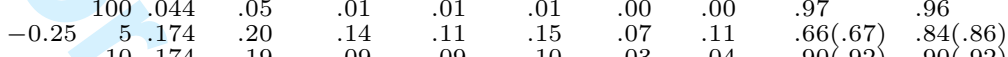

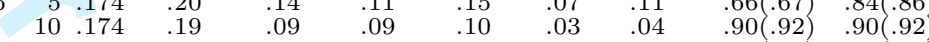

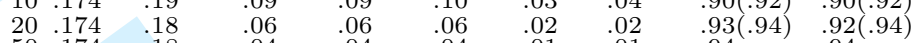

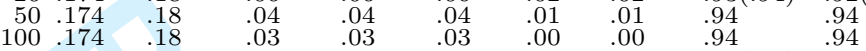

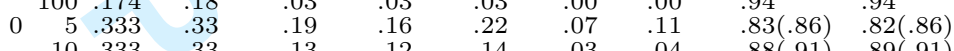

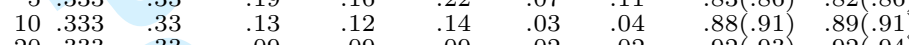

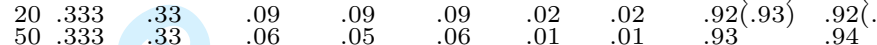

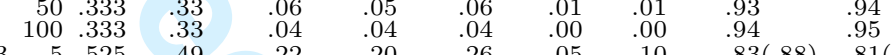

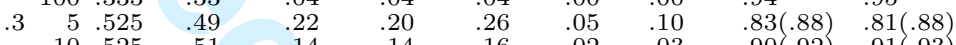

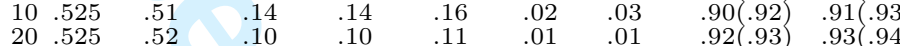

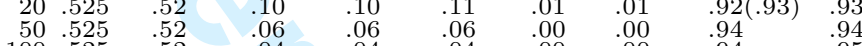

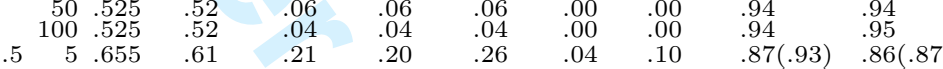

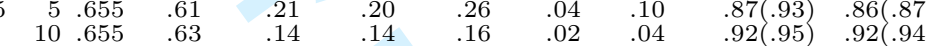

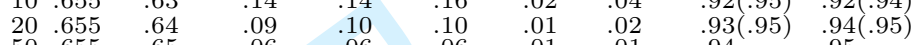

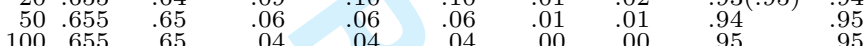

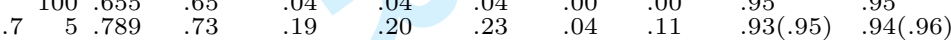

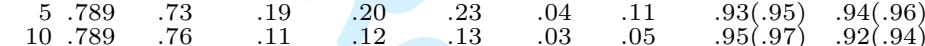

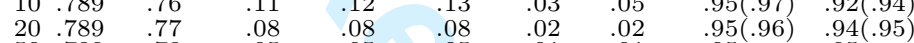

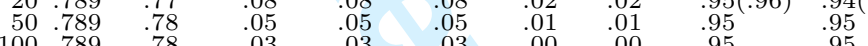

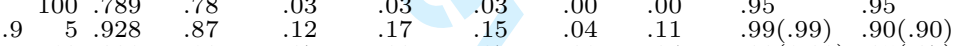

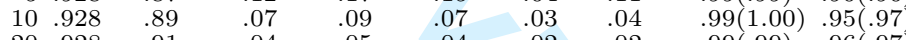

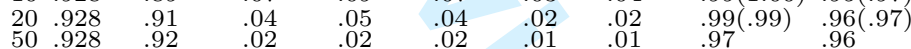

100.928

Table 1: Gaussian copula with equicorrelation with parameter $\theta$ for dimensions $d=3,5$ and 10 and sample sizes $n=5,10,20,50$ and 100. $\mathcal{W}_{0}$ denotes the theoretical value of Kendall's $\mathcal{W}$ for the parameter $\theta$ under consideration. $m($.$) and s($.$) denote mean and standard$ deviation of the respective quantity over $N=10000$ Monte Carlo replications. $\widehat{\sigma}^{B / J}$ refers to the bootstrap $(B, 500$ samples) and jackknife $(J)$ estimates of the standard deviation of $\widehat{\mathcal{W}}_{n}$. The last two columns display the simulated coverage probabilities $\mathrm{CP}^{B / J}$ for a nominal 95\% confidence interval for $\mathcal{W}_{0}$ using $\widehat{\sigma}^{B / J}$ and the Gaussian $95 \%$ quantile (and the $95 \%$ quantile of the $t_{\nu}$-distribution with $\nu=n-1$ degrees of freedom in brackets). 
Table 2: Clayton copula with parameter $\theta$ for dimensions $d=3,5$ and 10 and sample sizes $n=5,10,20,50$ and $100 . \mathcal{W}_{0}$ denotes the theoretical value of Kendall's $\mathcal{W}$ for the parameter $\theta$ under consideration. $m($.$) and s($.$) denote mean and standard deviation of the respective$ quantity over $N=10000$ Monte Carlo replications. $\widehat{\sigma}^{B / J}$ refers to the bootstrap $(B, 500$ samples) and jackknife $(J)$ estimates of the standard deviation of $\widehat{\mathcal{W}}_{n}$. The last two columns display the simulated coverage probabilities $\mathrm{CP}^{B / J}$ for a nominal $95 \%$ confidence interval for $\mathcal{W}_{0}$ using $\widehat{\sigma}^{B / J}$ and the Gaussian $95 \%$ quantile (and the $95 \%$ quantile of the $t_{\nu^{-}}$distribution with $\nu=n-1$ degrees of freedom in brackets). 


\subsection{Normal approximations to Kendall's $\mathcal{W}$}

The theorem in section 3.2 shows that $\widehat{\mathcal{W}}_{n}$ is asymptotically normally distributed under very weak conditions on the basic copula $C$. The simulation results in section 3.3 imply that the finite sample standard deviation of $\widehat{\mathcal{W}}_{n}$ can at least for $n \geq 20$ be satisfactorily estimated by the bootstrap or the jackknife. Even for $n=10$ and 5, they provide useful information on the scattering behavior of $\widehat{\mathcal{W}}_{n}$.

This section investigates how well the distribution of the standardized $\widehat{\mathcal{W}}_{n}$, i.e., of

$$
\frac{\widehat{\mathcal{W}}_{n}-\mathcal{W}_{0}}{\widehat{\sigma}_{\widehat{\mathcal{W}}_{n}}^{B / J}}
$$

can be approximated by a standard normal distribution, where $\widehat{\sigma}_{\widehat{\mathcal{W}}_{n}}^{B / J}$ is a bootstrap or jackknife estimator for the standard deviation of $\widehat{\mathcal{W}}_{n}$. For the Gaussian copula with equicorrelation and the Clayton copula, $N=10000$ Monte Carlo replications of the above statistic have been generated and kernel density estimates of its density have been plotted for various combinations of $n, d$ and parameters $\theta$. Eight of these graphs are shown in figures 1 and 2 ,

The results of the inspection of a large number of these graphs for all considered values of $d$ ( $n=5$ is not considered) can be summarized as follows:

- As expected, goodness of fit to the standard normal distribution increases if $n$ increases.

- Using the bootstrap estimates for the standard deviation leads to somewhat better fits than using the jackknife estimates, in particular for $n=10$ and 20. For $n=50$ and $n=100$, the difference is negligable.

- The fit is usually better for values of $\mathcal{W}_{0}$ near 0.5 than near the borders 0 or 1 . Indeed, values of $\mathcal{W}_{0}$ near 1 (near 0 ) imply a distribution of the normalized $\widehat{W}_{n}$ which is skewed to the right (skewed to the left), in particular for $n=10$ and $n=20$.

Approximate normality of $\widehat{\mathcal{W}}_{n}$ can be used to construct approximate $(1-\alpha)$ confidence intervals for $W_{0}$ via $\widehat{\mathcal{W}}_{n} \pm \Phi^{-1}(1-\alpha / 2) \widehat{\sigma}_{\widehat{\mathcal{W}}_{n}}^{B / J}$. The true probability of coverage was investigated 
Gauss, $d=5, n=20, w_{0}=0.05$ and $w_{0}=0.96$

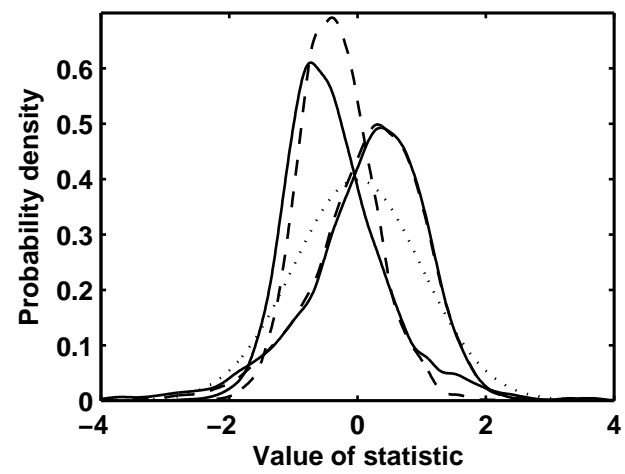

Gauss, $d=5, n=50, w_{0}=0.05$ and $w_{0}=0.96$

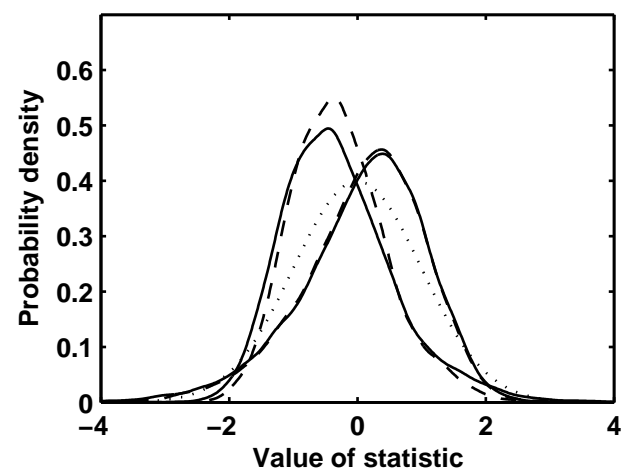

Gauss, $d=5, n=20, w_{0}=0.2$

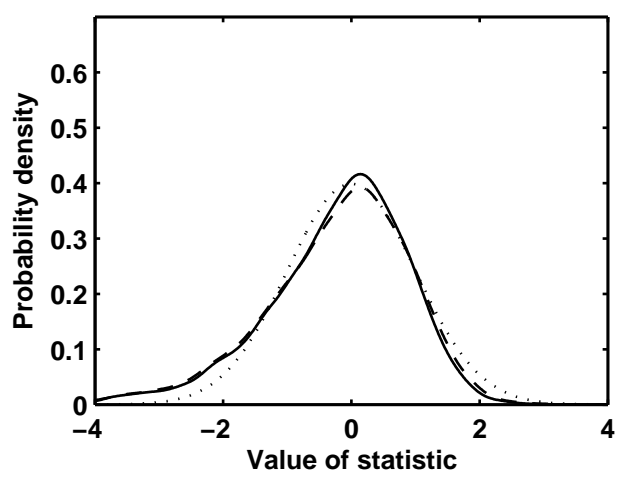

Gauss, $d=5, n=50, w_{0}=0.2$

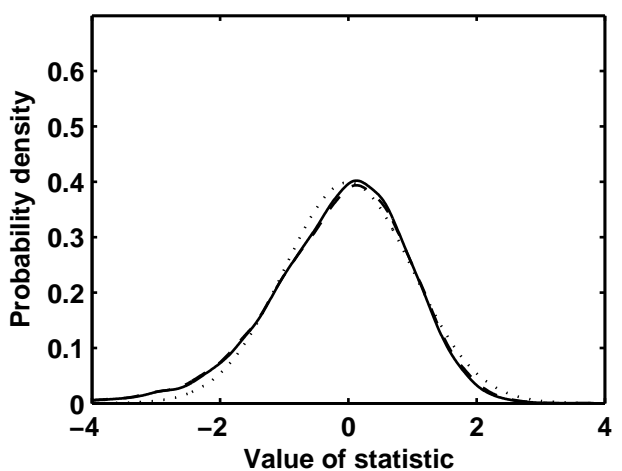

Figure 1: Gaussian copula. Kernel density estimates of $\left(\widehat{\mathcal{W}}_{n}-\mathcal{W}_{0}\right) / \widehat{\sigma}_{\widehat{\mathcal{W}}_{n}}^{B / J}$ using the bootstrap $(B$, solid line) and jackknife $(J$, dashed line) estimates of the standard deviation of $\widehat{\mathcal{W}}_{n}$. Left skewed densities refer to smaller values of $\mathcal{W}_{0}$. The dotted line depicts the density of the standard normal distribution.

for a nominal coverage probability of $1-\alpha=0.95$ by means of simulation. The results are displayed in the last two columns of tables 1 and 2 for various combinations of parameters.

The procedure works satisfactorily for $n=50$ and 100. For $n=5$ and 10 and 20, there are instances where the true confidence level is too low, in particular for $d=5$ and $d=10$ and for values of $\mathcal{W}_{0}$ near $1 / d$, i.e., the independence case. In those cases the coverage may be improved by using quantiles of the $t$-distribution instead of Gaussian quantiles when constructing the confidence intervals. Therefore, for $n=10$ and $n=20$ the tables also show 

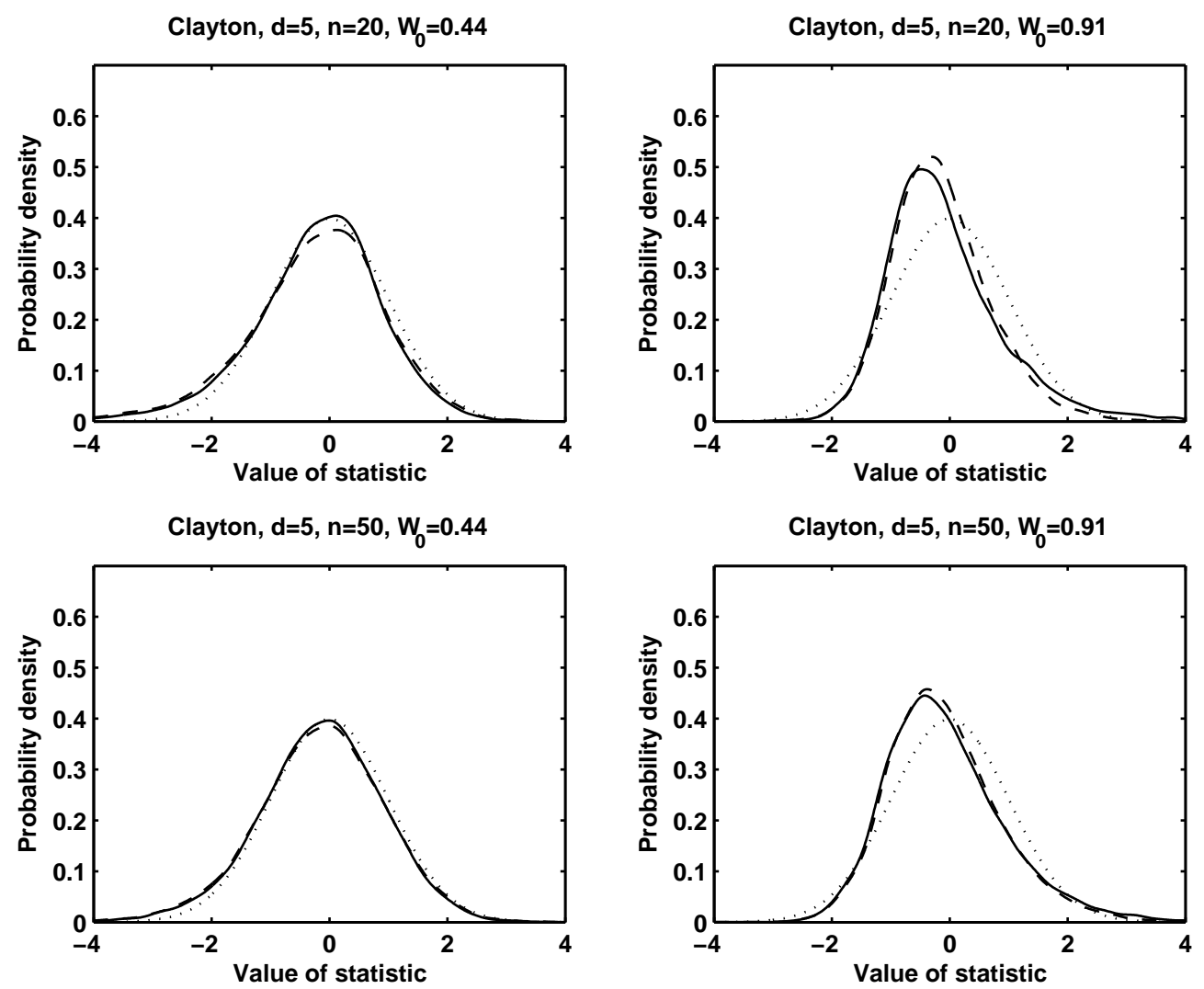

Figure 2: Clayton Copula. Kernel density estimates of $\left(\widehat{\mathcal{W}}_{n}-\mathcal{W}_{0}\right) / \widehat{\sigma}_{\widehat{\mathcal{W}}_{n}}^{B / J}$ using the bootstrap ( $B$, solid line) and jackknife $(J$, dashed line) estimates of the standard deviation of $\widehat{\mathcal{W}}_{n}$. Left skewed densities refer to smaller values of $\mathcal{W}_{0}$. The dotted line depicts the density of the standard normal distribution.

the true coverage probabilities of confidence intervals based on $t$-distributions with $\nu=n-1$ degrees of freedom (values in brackets). As to be expected, coverage probabilities are higher now, leading to a better agreement with the nominal coverage rates for values of $\mathcal{W}_{0}$ around $1 / 2$.

The poor fit to the normal distribution for small values of $n$ calls for the exact small sample distribution of $\widehat{\mathcal{W}}_{n}$. In the case of independence of the rankings, this distribution has been derived by Kendall and Babington Smith (1939). In the 
dependence case, however, this distribution is not known. Our results on the asymptotic distribution of $\widehat{\mathcal{W}}_{n}$ suggest that the finite sample distribution of $\widehat{\mathcal{W}}_{n}$ depends on the copula $C$ of the rankings and not only on $\mathcal{W}_{0}$. The relationship between $C$ and the finite sample distribution, however, seems to be extremely complicated. It remains an open problem if at least some transformation of $\widehat{\mathcal{W}}_{n}$ may lead to a better fit to the Gaussian density.

\section{Application to financial data}

Until recently, the dependence in financial data has usually been measured by the Pearson coefficient of correlation. The increasing use of copulas in modelling and analyzing financial data, however, entails the use of copula-based measures of dependence and association. For a critical discussion of the related issues, in particular the many shortcomings of Pearson's coefficient of correlation, see Embrechts et al. (2002). In finance, the strength of association of asset returns (as measured by $\mathcal{W}$ ) and its development over time provide useful information on the amount of diversification contained in a portfolio of assets. A value of $\mathcal{W}$ near 1 indicates closeness to comonotonicity and poor diversification properties of the portfolio. A review of comonotonicity in finance, actuarial sciences and, in particular, in risk management is given in Dhaene et al. $(2000 a, b)$. Besides asset returns, volatilities of assets play a crucial role in the valuation of corresponding derivatives (such as put or call options). Association of volatilities and its development over time is therefore an interesting indicator for the derivatives market. In the following, we will apply Kendall's $\mathcal{W}$ to investigate returns and volatilities of the DAX-30 assets.

Let $\mathbf{X}=\left(X_{1}, \ldots, X_{30}\right)$ denote daily returns of these assets and let $\mathbf{X}_{1}, \ldots, \mathbf{X}_{250}$ denote observations on $\mathbf{X}$ on 250 consecutive trading days. From these observations, the $30 \times 250$ matrix of ranks $\mathbf{R}$ of the returns, the empirical value $\widehat{\mathcal{W}}_{250}$ and the bootstrap and jackknife estimates $\widehat{\sigma}^{B}$ and $\widehat{\sigma}^{J}$ are computed as in section 3, This is conducted for a moving window 
over a data set from 2nd of January 2003 to 6 th of November 2009. The values of $\widehat{\mathcal{W}}_{250, t}$, where $t$ denotes the final day included in the window, are displayed in figure 3 , The confidence intervals are computed as in section 3 . In the same way $\widehat{\mathcal{W}}_{250, t}$ is computed for the volatilities $\left|X_{1}\right|, \ldots,\left|X_{30}\right|$. Both values are compared in figure 4. The width of the $95 \%$ confidence intervals is about 0.078 for Kendall's $\mathcal{W}$ for the returns and 0.091 for the volatilities.

Inspection of both figures reveals significant changes of Kendall's $\mathcal{W}$ for returns and volatilities over time. This is particularly the case for the volatilities which are between 0.15 and 0.37 with a remarkable increase since the beginning of 2006. Association of returns is between 0.32 and 0.45 and is always higher than those of the volatilities. The moving pattern of both series is essentially the same. Volatilities, however, seem to react somewhat stronger than returns.

Estimating Kendall's $\mathcal{W}$ over the entire time period from January 2nd 2003 to November 6th 2009 (i.e., $n=1787$ observations) results in

$$
\begin{array}{lll}
\widehat{\mathcal{W}}_{n}=0.40 & \widehat{\sigma}_{\mathcal{W}_{n}}=0.009 & \text { for returns } \\
\widehat{\mathcal{W}}_{n}=0.31 & \widehat{\sigma}_{\mathcal{W}_{n}}=0.010 & \text { for volatilities }
\end{array}
$$

Comparing these results to figure 3 , the need to model financial data adaptively by taking into account the permanent change in the strength of association in the underlying assets, becomes evident.

\section{Conclusion}

Kendall's $\mathcal{W}$ is a measure of agreement between $d$ rankings of $n$ objects. Copula theory enables to investigate $\mathcal{W}$ for the non-null case, i.e., the case when the rankings are not independent, in a very general way. It is possible to define a population version $\mathcal{W}_{0}$ of Kendall's $\mathcal{W}$ in a straightforward manner. Further, it can be shown that the related estimator $\widehat{\mathcal{W}}_{n}$ for $\mathcal{W}_{0}$ is asymptotically normally distributed under weak assumptions. Its standard deviation can be determined by the bootstrap and the jackknife in a reliable way for $n \geq 10$. The standardized version of $\widehat{\mathcal{W}}_{n}$ can be approximated by the normal distribution at least for 


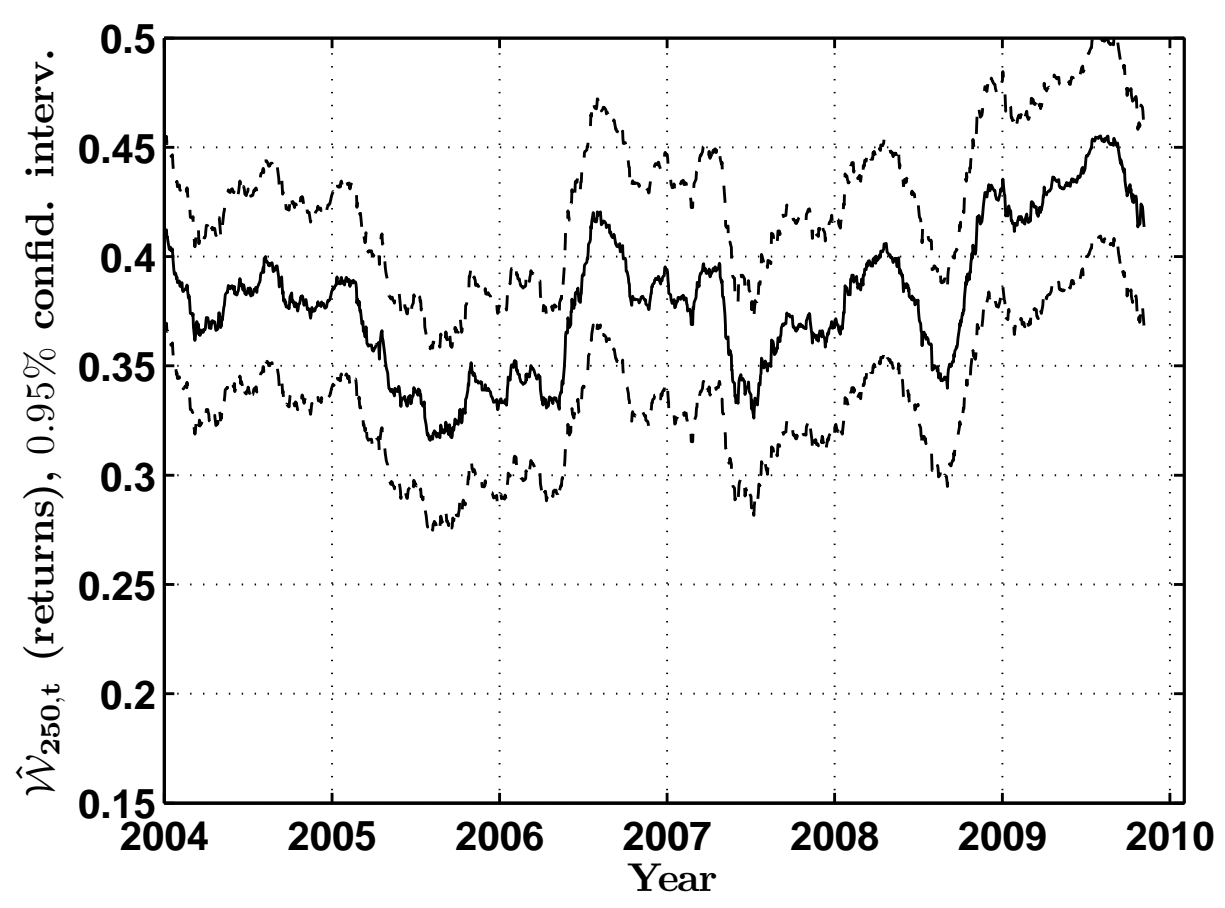

Figure 3: Kendall's $\mathcal{W}$ for returns with corresponding $95 \%$ confidence interval for the DAX data. The estimates rely on moving windows of 250 trading days. For the confidence intervals, bootstrap estimates of the variance are used as described in section 3 .

$n \geq 20$ if $\mathcal{W}_{0}$ is not too close to 0 or 1 , i.e., the cases of complete disagreement and agreement of the rankings. A suitable transformation of $\widehat{\mathcal{W}}_{n}$ might be implemented to remove skewness in these cases but it seems to be an open problem which transformation can serve for this purpose for a broad range of copulas.

\section{Appendix: Proofs}

The proof of the theorem relies on the results in Schmid and Schmidt (2007). These authors have shown that under the assumption of the theorem we have

$$
\sqrt{n}\left(\widehat{\bar{\rho}}_{S, n}-\bar{\rho}_{S}\right) \stackrel{w}{\longrightarrow} Z \sim N\left(0, \sigma^{2}\right)
$$




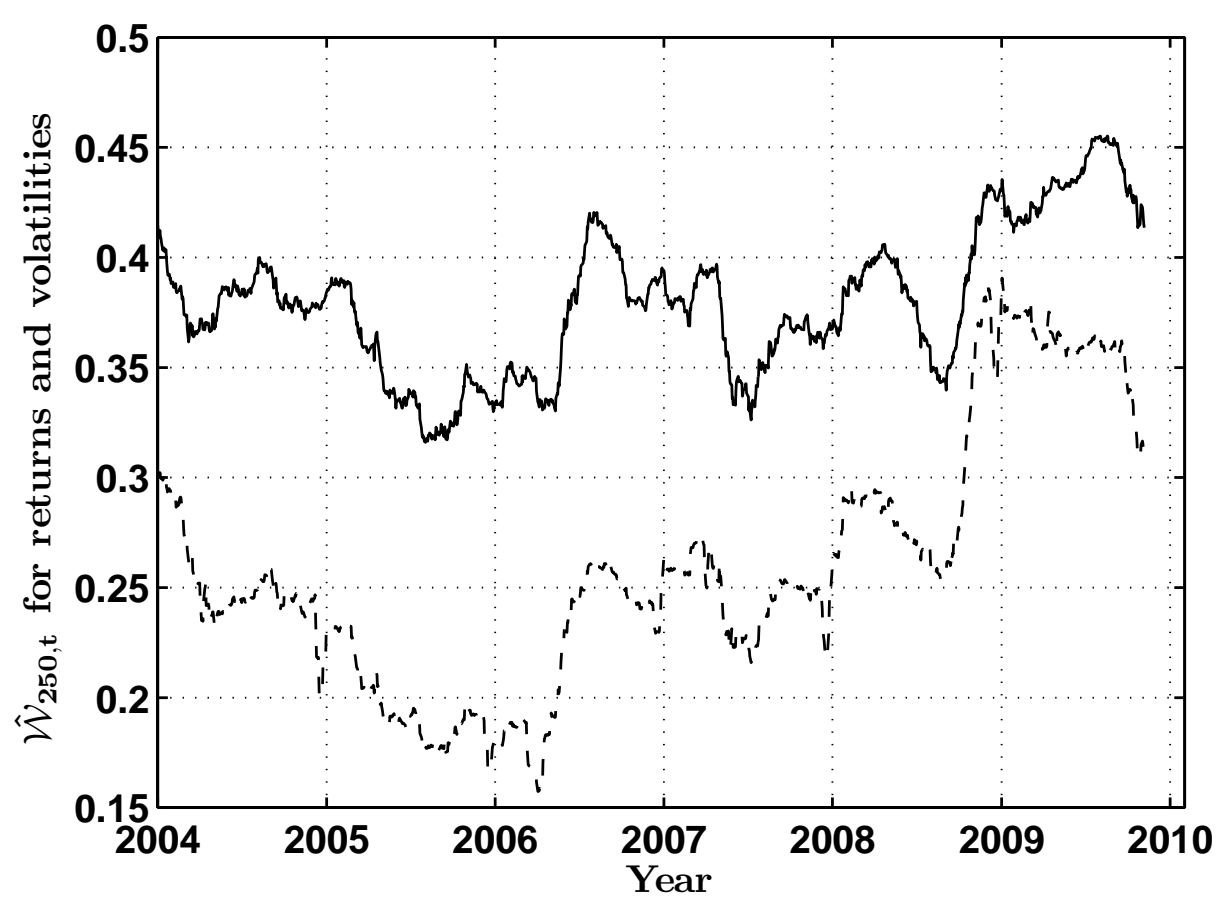

Figure 4: Kendall's $\mathcal{W}$ for returns (solid line) and volatilities (dashed line) for the DAX data. The estimates rely on moving windows of 250 trading days.

where

$$
\sigma^{2}=144\left(\begin{array}{l}
d \\
2
\end{array}\right)^{-2}\left[\sum_{\substack{k<l \\
s<t}} \int_{0,1]^{2}} \int_{[0,1]^{2}} \mathrm{E}\left[\mathbb{G}_{C}\left(\mathbf{u}^{(k, l)}\right) \mathbb{G}_{C}\left(\mathbf{v}^{(s, t)}\right)\right] d\left(u_{k}, u_{l}\right) d\left(v_{s}, v_{t}\right)\right]
$$

Because of

$$
W_{0}=\left(\frac{d-1}{d}\right) \bar{\rho}_{S}+\frac{1}{d}
$$

and

$$
\widehat{\mathcal{W}}_{n}=\left(\frac{d-1}{d}\right) \widehat{\bar{\rho}}_{S, n}+\frac{1}{d}
$$

the result of the theorem follows. 
In case of independence, i.e., $C=\Pi$, Schmid and Schmidt (2007) derived that

$$
\sqrt{n}\left(\widehat{\bar{\rho}}_{S, n}-0\right) \stackrel{w}{\longrightarrow} N\left(0, \sigma^{2}\right)
$$

where

$$
\sigma^{2}=\left(\begin{array}{l}
d \\
2
\end{array}\right)^{-1}
$$

Therefore

$$
\sqrt{n}\left(\widehat{\mathcal{W}}_{n}-\frac{1}{d}\right) \stackrel{w}{\longrightarrow} N\left(0, \sigma_{\Pi}^{2}\right)
$$

where

$$
\sigma_{\Pi}^{2}=\left(\frac{d-1}{d}\right)^{2}\left(\begin{array}{l}
d \\
2
\end{array}\right)^{-1}=\frac{2(d-1)}{d^{3}}=\frac{2}{d}\left(\frac{1}{d}\left(1-\frac{1}{d}\right)\right) .
$$

\section{References}

Cherubini, U., Luciano, B., Vecchiato, W. (2004). Copula Methods in Finance. New York: John Wiley \& Sons.

Clayton, D. (1978). A model for association in bivariate life tables and its application in epidemiological studies of familial tendency in chronic disease incidence. Biometrika 65:141151.

Dhaene, J., Denuit, M., Goovaerts, M. J., Kaas, R., Vyncke, D. (2002a). The concept of comonotonicity in actuarial science and finance: applications. Insurance: Mathematics and Economics 31:33-161.

- (2002b). The concept of comonotonicity in actuarial science and finance: theory. Insurance: Mathematics and Economics 31:3-33.

Embrechts, P., McNeil, A., Straumann, D. (2002). Correlation and dependency in risk management: properties and pitfalls. In: Dempster, M. A. H. ed., Risk Management: Value at Risk and Beyond. Cambridge: Cambridge University Press. 
Genest, C., Quessy, J.-F., Rémillard, B. (2007). Asymptotic local efficiency of Cramér-von Mises tests for multivariate independence. Annals of Statistics 35:166-191.

Genest, C., Rémillard, B. (2004). Tests of independence and randomness based on the empirical copula process. Test 13:335-369.

Joe, H. (1997). Multivariate Models and Dependence Concepts. London: Chapman \& Hall.

Kendall, M. G., Babington Smith, S. B. (1939). The problem of $m$ rankings. The Annals of Mathematical Statistics 10:275-287.

Kendall, M. G., Gibbons, J. D. (1990). Rank Correlation Methods. London: Edward Arnold.

Kraemer, H. C. (1976). The small sample nonnull properties of Kendall's coefficient of concordance for normal populations. Journal of the American Statistical Association 71:608-613.

Loretta, L., Schucany, W. R. (1975). Some properties of a test for concordance of two groups of rankings. Biometrika 62:417-423.

Mallows, C. L. (1957). Non-null ranking models. I. Biometrika 44:114-130.

Nelsen, R. B. (2006). An Introduction to Copulas. New York: Springer.

Palachek, A., Schucany, W. (1984). On approximate confidence intervals for measures of concordance. Psychometrika 49:133-141.

Schmid, F., Schmidt, R. (2007). Multivariate extensions of Spearman's rho and related statistics. Statistics \& Probability Letters 77:407-416.

Shao, J., Dongsheng, T. (1995). The Jackknife and Bootstrap. New York: Springer.

Sklar, A. (1959). Fonctions de répartition à $n$ dimensions et leurs marges. Publications de l'Institut de Statistique de l'Universite de Paris 8:229-231. 